\title{
Diretrizes
}

\section{Doença arterial coronariana}

A doença arterial coronariana (DAC) é a primeira causa de óbito nas sociedades modernas ${ }^{1}$ e a ecocardiografia tem importante papel na avaliação de pacientes com suspeita clínica ou comprovada desta doença.

\section{1 - Síndrome coronariana aguda}

Quando o miocárdio torna-se isquêmico, rapidamente ocorre alteração na função regional, caracterizada por mudanças no movimento e espessamento da parede ventricular, podendo se tornar hipocinética, acinética ou discinética. Estas alterações são proporcionais à gravidade da doença e da duração da isquemia.

A fim de normatizar a avaliação da função regional para as várias modalidades de imagem cardiovascular, Cerqueira e cols ${ }^{1}$, utilizaram um modelo de divisão do VE em 17 segmentos e atribuiram escores de função segmentar emtre 1-4 (escore 1 = contração normal, 2 = hipocinesia, 3 = acinesia e $4=$ discinesia). Do escore atribuído aos segmentos analisados, obtém-se uma média conhecida como índice de escore de motilidade e espessamento de parede (IEMP). Atualmente, sabe-se que o IEMP apresenta uma relação direta com a funçãodo VE. Seu valor normal é igual a 1 e valores $\geq 2$, se correlacionam com disfunção importante do VE e com maior probabilidade para a ocorrência de arritmias graves, insuficiência cardíaca e morte ${ }^{2}$.

Além do movimento, o espessamento sistólico do segmento miocárdico expressa de forma mais precisa a motilidade do VE. A ecocardiografia pode confirmar ou afastar alterações segmentares da motilidade que sugerem, no momento da dor, a presença de isquemia miocárdica aguda, infarto agudo do miocárdio (IAM) ou mesmo infarto pregresso ${ }^{3,4}$.

A avaliação da espessura diastólica e refrigência de cada segmento miocárdico, na condição de repouso, sugere presença ou ausência de viabilidade miocárdica, embora em algumas condições se imponham a complementação diagnóstica com outros métodos 5 .

Além da análise do movimento, espessura e ecogenicidade da parede regional do VE, a avaliação da função sistólica, diastólica, dos fluxos intracardíacos, vasos da base e do VD são de grande valor na estratificação de pacientes com síndromes coronarianas agudas e no diagnóstico diferencial de outras afecções comumente confundidas ou que podem coexistir na presença de DAC como a estenose aórtica grave, embolia pulmonar, dissecção de aorta, pericardite e presença de tumores cardíacos.

A presença de alteração do movimento da parede regional do VE pode ocorrer em doenças cardíacas não isquêmicas, como doença de Chagas, forma assimétrica da cardiomiopatia hipertrófica, movimento dissincrônico do septo interventricular por bloqueio de ramo esquerdo, sobrecarga volumétrica do VD, síndrome de Wolff-Parkinson-White, pós operatório de cirurgia cardíaca e na presença de marcapasso endocárdico. Excetuando a alteração de motilidade encontrada na doença de Chagas, todas as demais podem ser diferenciadas das anormalidades isquêmicas pela presença de espessamento sistólico preservado.
Quando há hipocontratilidade de todas as paredes do VE devido à DAC multiarterial, o diagnóstico diferencial com cardiomiopatia dilatada de origem não isquêmica pode ser difícil. A presença de cavidade ventricular esquerda pouco dilatada, segmentos miocárdicos mais refringentes e finos que outros podem sugerir etiologia isquêmica, porém nenhuma dessas alterações é significativamente específica.

\subsection{1 - Ecocardiografia sob estresse na síndrome coronariana aguda}

A ecocardiografia sob estresse tem sido utilizada em pacientes admitidos na unidade de emergência com dor torácica, ECG não diagnóstico, enzimas cardíacas normais e suspeita de angina instável de risco baixo ou intermediário. Tradicionalmente, a ecocardiografia sob estresse é realizada pelo menos 24 horas após a melhora da dor, porém seu uso precoce, entre 6 a 12 horas foi validado sem risco de complicações significativas. O ideal para seu uso é definir a presença ou não de isquemia, possibilitando alta hospitalar precoce nos casos com teste negativo, uma vez que, apresenta alto valor preditivo negativo ${ }^{6}$. O estresse pode ser físico ou farmacológico (dobutamina ou dipiridamol associados à atropina), dependendo da capacidade do paciente de realizar esforço físico e experiência do serviço. Além da presença de isquemia, o ecocardiograma sob estresse permite estratificar a gravidade anatomo-funcional da DAC subjacente.

Em pacientes hospitalizados com IAM não complicado, a ecocardiografia sob estresse farmacológico é segura e útil na estratificação de risco não invasiva. Pacientes com exames eficazes e provas negativas ou com pequenas áreas isquêmicas apresentam bom prognóstico no médio prazo ${ }^{7}$.

\subsection{2 - Ecocardiografia com contraste na síndrome coronariana aguda}

Na fase aguda do infarto, o uso do contraste ecocardiográfico tem importante papel, melhorando a definição das bordas das paredes do VE e analisando a perfusão miocárdica.

O uso do contraste para avaliar a borda endocárdica (avaliação da função global e regional) se aplica quando pelo menos dois segmentos miocárdicos não são visualizados ou o são inadequadamente, conforme estabelecido nas diretrizes da AHA, e a má visualização de quatro segmentos miocárdicos ao corte apical implica em indicação do uso de contraste, segundo as diretrizes latino-americanas ${ }^{8,9}$.

Vários estudos demonstram que em cerca de 10\%-20\% dos casos a análise da borda endocárdica é subótima e há evidências de que o uso de contraste aperfeiçoa a imagem, tanto fundamental quanto com harmônica. ${ }^{10,11}$.Nesse caso, o propósito é de visualizar o agente de contraste dentro da cavidade do VE e não no miocárdio, definindo melhor o espessamento e motilidade parietal. Assim, a administração do contraste é capaz de converter um exame não diagnóstico em diagnóstico em 75\% dos casos e aumentar a capacidade diagnóstica do método para lesões univasculares ${ }^{12}$.

Em duas situações da prática clínica, o uso do contraste oferece grandes vantagens na imagem, com um mínimo 


\section{Tabela 22 - Recomendações do ecocardiograma na DAC aguda}

\begin{tabular}{lc}
\hline Recomendações & Classe \\
\hline Angina instável com instabilidade hemodinâmica & । \\
\hline $\begin{array}{l}\text { Suspeita de complicações mecânicas no IAM (aneurisma de VE, } \\
\text { rotura de parede livre, de septo interventricular ou de músculo } \\
\text { papilar, derrame pericárdico) }\end{array}$ & lb \\
\hline $\begin{array}{l}\text { No IAM de parede inferior, avaliação da possibilidade do } \\
\text { envolvimento do VD }\end{array}$ & । \\
\hline $\begin{array}{l}\text { Auxílio no diagnóstico diferencial de EAo grave, embolia pulmonar, } \\
\text { dissecção de aorta*, pericardites e presença de tumores cardíacos }\end{array}$ & । \\
\hline $\begin{array}{l}\text { Durante a dor de possível origem isquêmica, com ECG e enzimas } \\
\text { cardíacas não conclusivas }\end{array}$ & Ilb \\
\hline $\begin{array}{l}\text { Identificação da localização e gravidade da DAC em pacientes sob } \\
\text { vigência de isquemia miocárdica aguda }\end{array}$ & \\
\hline
\end{tabular}

*ETE tem maior acurácia e pode fornecer informações adicionais àquelas obtidas por meio da ecocardiografia transtorácica.

de aumento no tempo de exame: na unidade de terapia intensiva, onde as imagens são limitadas, a obtenção da FE e da função regional torna-se bem superior com o contraste ${ }^{13}$; e durante a ecocardiografia sob estresse, onde a presença de doença pulmonar, obesidade, hiperventilação e taquicardia podem resultar em imagens não diagnósticas em 20\%-30\% dos pacientes.

A recanalização da artéria relacionada ao infarto (ARI) com fluxo TIMI grau 3 não é sinônimo de perfusão microvascular ${ }^{14}$. O fenômeno de no reflow (não restabelecimento do fluxo tecidual) é demonstrado pela ecocardiografia com contraste como áreas do miocárdio sem contraste, mesmo após abertura da ARI. Tal fenômeno indica lesão miocárdica irreversível e está relacionado à disfunção e remodelamento ventricular ${ }^{15,16}$.

\section{Referências}

1. Cerqueira MD, Weissman NJ, Dilsizian V, Jacobs AK, Kaul S, Laskey WK, et al. Standardized myocardial segmentation and nomenclature for tomographic imaging of the heart: a statement for healthcare professionals from the Cardiac Imaging Committee of the Council on Clinical Cardiology of the American Heart Association. Circulation. 2002; 105: 539-42.

2. Picano E. Stress echocardiography. 2th. ed. Berlin (Heidelberg): SpringerVerlag; 1994

3. Sabia P, Afrookteh A, Touchstone DA, Keller MW, Esquivel L, Kaul S. Value of regional wall motion abnormality in the emergency room diagnosis of acute myocardial infarction: a prospective study using two-dimensional echocardiography. Circulation. 1991; 84 (3): 185-92.

4. Kontos MC, Arrowood JA, Paulsen WH, Nixon JV. Early echocardiography can predict cardiac events in emergency department patients with chest pain. Ann Emerg Med. 1998; 31: 550-7.

5. Cwajg JM, Cwajg E, Nagueh SF, He ZX, Qureshi U, Olmos LI, et al, End-diastolic wall thickness as a predictor of recovery of function in myocardial hibernation: relation to rest-redistribution TI-201 tomography and dobutamine stress echocardiography. J Am Coll Cardiol. 2000; 35: 1152-61.
Tabela 23 - Recomendações da ecocardiografia sob estresse na síndrome coronariana aguda

\begin{tabular}{lc}
\hline Recomendações & Classe \\
\hline $\begin{array}{l}\text { Pacientes com angina instável de risco baixo ou intermediário } \\
\text { controlada clinicamente }\end{array}$ & \|la \\
\hline $\begin{array}{l}\text { Para avaliar significado funcional de obstrução coronária moderada } \\
\text { à angiografia, desde que o resultado interfira na conduta }\end{array}$ & \|la \\
\hline Estratificação de risco após o infarto do miocárdio não complicado & \|la \\
\hline $\begin{array}{l}\text { Estratificação de risco na angina instável após 48 horas de } \\
\text { estabilização }\end{array}$ & \|lb \\
\hline $\begin{array}{l}\text { Angina instável de alto risco ou infarto agudo do miocárdio } \\
\text { complicado }\end{array}$ & IIl \\
\hline
\end{tabular}

Tabela 24 - Recomendações da ecocardiografia com contraste por microbolhas na DAC aguda

\begin{tabular}{lc}
\hline Recomendações & Classe \\
\hline $\begin{array}{l}\text { Delineamento de bordas endocárdicas em pacientes com janela } \\
\text { subótima em repouso }\end{array}$ & । \\
\hline $\begin{array}{l}\text { Melhora do sinal Doppler do fluxo aórtico em pacientes com sinal } \\
\text { inadequado e suspeita de EAo importante associada }\end{array}$ & । \\
\hline $\begin{array}{l}\text { Delineamento de bordos endocárdicos durante a ecocardiografia } \\
\text { sob estresse em pacientes com imagem subótima em repouso }\end{array}$ & IIb \\
\hline $\begin{array}{l}\text { Avaliação da perfusão miocárdica em pacientes com suspeita de } \\
\text { IAM e ECG não diagnóstico }\end{array}$ & III \\
\hline Avaliação da perfusão miocárdica em todos os tipos de IAM & III \\
\hline
\end{tabular}

6. Geleijnse ML, Elhendy A, Kasprzak JD, Rambaldi R, Van Domburg RT Cornel JH, et al. Safety and prognostic value of early dobutamine-atropine stress echocardiography in patients with spontaneous chest pain and a non-diagnostic electrocardiogram. Eur Heart J. 2000; 21: 397-406.

7. Picano E, Sicari R, Raciti M, Pingitore A, Vassalle C, Mathias W, et al. Role of stress echocardiography in risk stratification early after an acute myocardial infarction. EPIC (Echo Persantin International Cooperative) and EDIC (Echo Dobutamine International Cooperative) Study Groups. Eur Heart J. 1997; 18: 78-85.

8. Mulvagh SL, Rakowski H, Vannan MA, Abdelmoneim SS, Becher H, Bierig SM, et al. American Society of Echocardiography Consensus Statement on the Clinical Applications of Ultrasonic Contrast Agents in Echocardiography. J Am Soc Echocardiogr. 2008; 21 (11): 1179-201.

9. Ronderos R, Morcerf F, Boskis M, Corneli D, Cuenca G, Gutiérrez F, et al. Diretriz e recomendações para o uso da ecocardiografia contrastada: Fórum Latino-Americano de Ecocardiografia com Contraste. Arq Bras Cardiol. 2007; 88 (2): 1-12.

10. Cohen JL, Cheirif J, Segar DS, Gillam LD, Gottdiener JS, Hausnerova E, et al Improved left ventricular endocardial border delineation and opacification 


\section{Diretrizes}

with OPTISON, a new echocardiographic contrast agent. Results of a phase III multicenter trial. J Am Coll Cardiol. 1998; 32 (3): 746-52.

11. Mulvagh SL, Rainbird AJ, Al-Mansour HA. Harmonic dobutamine stress echocardiography: does contrast make a difference in diagnostic feasibility and accuracy? Circulation. 1998; 12: 527-63.

12. Dolan MS, Riad K, El-Shafei A, Puri S, Tamirisa K, Bierig M, et al. Effect of intravenous contrast for left ventricular opacification and border definition on sensitivity and specificity of dobutamine stress echocardiography compared with coronary angiography in technically difficult patients. Am Heart J. 2001; 142: 908-15.

13. Kornbluth M, David H, Brown P. Contrast echocardiography is superior to tissue harmonics for assessment of left ventricular function in mechanically ventilated patients. Am Heart J. 2000; 140: 291-6.

\section{2 - Síndrome coronariana crônica}

A ecocardiografia constitui importante subsídio em pacientes com DAC crônica, fornecendo informações sobre função ventricular, isquemia e viabilidade miocárdica, além de diagnosticar outras alterações associadas.

\subsection{1 - Ecocardiografia sob estresse na DAC crônica}

A ecocardiografia sob estresse é um método não invasivo e estabelecido para avaliação de pacientes com DAC suspeita ou conhecida, exercendo um importante papel na determinação do seu diagnóstico e prognóstico. O estresse cardiovascular causa isquemia miocárdica em regiões supridas por uma artéria com grau significativo de estenose, fenômeno manifestado por alteração transitória da contração segmentar ${ }^{1}$.

Os métodos disponíveis para a indução do estresse são: o esforço físico (esteira ou bicicleta ergométrica) ou farmacológico (dobutamina, dipiridamol e adenosina), além da estimulação atrial transesofágica. A ecocardiografia sob estresse apresenta boa acurácia para detecção de isquemia miocárdica induzida ${ }^{1}$ em pacientes com probabilidade préteste intermediária ou alta.

\section{Tabela 25 - Recomendações da ecocardiografia transtorácica na} DAC crônica

\begin{tabular}{lc}
\hline Recomendações & Classe \\
\hline Avaliação inicial da função de VE & I \\
\hline $\begin{array}{l}\text { Avaliação da função do VE quando há sinais de ICC ou com } \\
\text { mudança do quadro clínico ou exame físico }\end{array}$ & I \\
\hline $\begin{array}{l}\text { Suspeita de complicações, como pseudoaneurismas, aneurismas } \\
\text { e insuficiência mitral }\end{array}$ & III \\
\hline $\begin{array}{l}\text { Avaliação inicial de assintomáticos com baixa probabilidade de DAC } \\
\text { Reavaliação periódica rotineira de pacientes estáveis sem } \\
\text { mudança na terapia }\end{array}$ & III \\
\hline
\end{tabular}

14. Ito H, Tomooka T, Sakai N, Yu H, Higashino Y, Fujii K, et al. Lack of myocardial perfusion immediately after successful thrombolysis: a predictor of poor recovery of left ventricular function in anterior myocardial infarction. Circulation. 1992; 85: 1699-705.

15. Ito H, Maruyama A, Iwakura K, Takiuchi S, Masuyama T, Hori M, et al. Clinical implications of the no reflow phenomenon: a predictor of complications and left ventricular remodeling in reperfused anterior wall myocardial infarction. Circulation. 1996; 93: 223-8.

16. Caldas MA, Tsutsui JM, Kowatsch I, Andrade JL, Nicolau JC, Ramires JF, et al. Value of myocardial contrast echocardiography for predicting left ventricular remodeling and segmental functional recovery after anterior wall acute myocardial infarction. J Am Soc Echocardiogr. 2004; 17: 923-32.

Quando comparada ao teste ergométrico, a ecocardiografia sob estresse tem maior sensibilidade e especificidade para o diagnóstico de DAC e apresenta valor clínico adicional na detecção e localização da isquemia miocárdica, com características e resultados similares ao $\mathrm{SPECT}^{2}$. A adição de atropina ao eco estresse farmacológico aumenta a acurácia do teste e diminui o número de exames ineficazes, especialmente sob uso de betabloqueador ${ }^{3}$. Um teste negativo está associado a baixo risco de eventos cardiovasculares durante o acompanhamento, sendo menor que $1 \%$ em um ano ${ }^{4}$. E um teste positivo implica em risco anual elevado de eventos.

Em pacientes com DAC crônica estabelecida, a função contrátil miocárdica pode estar deprimida devido à necrose miocárdica ou ao miocárdio hibernado; nestes casos, há indicação para análise de viabilidade miocárdica. Assim, em pacientes com DAC multiarterial e função de VE deprimida, a melhora da contração miocárdica segmentar durante baixas doses de dobutamina é considerada indicativa de reserva contrátil e é preditora de melhora da função ventricular após cirurgia de revascularização miocárdica ${ }^{5}$. A ecocardiografia de estresse também apresenta grande valor na estratificação de risco pré-operatório em pacientes submetidos à cirurgia vascular. Um exame normal apresenta alto valor preditivo negativo (93\%-100\%) para eventos cardiovasculares, sendo que um teste negativo dispensa outras investigações ${ }^{6}$.

\subsection{2 - Utilização do contraste ecocardiográfico na DAC crônica}

O uso de contraste ecocardiográfico por microbolhas possibilita uma melhor definição dos bordos endocárdicos, permitindo uma avaliação mais adequada do espessamento parietal e da função contrátil global e segmentar do VE, em repouso e sob estresse ${ }^{7}$.

Embora a ecocardiografia sob estresse tenha alta sensibilidade e especificidade na detecção de isquemia miocárdica, pode haver redução na definição da imagem no pico do estresse, o que aumenta a variabilidade intra e 
interobservador ${ }^{8}$. Isso pode ser compensado de modo eficaz e seguro, com o uso do contraste para ultrassom ${ }^{9}$.

Os agentes de contraste podem ser utilizados em qualquer modalidade de estresse ecocardiográfico. O Optimize Trial, estudo multicêntrico duplo cego, mostrou que a visualização adequada dos segmentos aumentou de $72 \%$ para $95 \%$ com o uso do contraste; e sob estresse, a diferença aumentou de $67 \%$ para $96 \%$, sem e com contraste, respectivamente ${ }^{10}$.

\subsection{3 - Avaliação da perfusão miocárdica e do fluxo coronariano}

A análise da perfusão miocárdica tem basicamente dois objetivos: avaliar isquemia e viabilidade, e é considerada um método adicional e complementar às modalidades existentes. Há duas situações clínicas em que o método tem sido validado: no IAM e na detecção de estenose coronariana.

A detecção não invasiva da DAC tem sido realizada principalmente pela ecocardiografia de estresse e pela medicina nuclear. A ecocardiografia de estresse associada ao uso de contraste permite a avaliação simultânea da contração e perfusão miocárdicas, detectando a repercussão funcional da DAC. Para a análise da perfusão é importante a adição de um vasodilatador coronariano (dipiridamol, adenosina ou análogos), apesar da dobutamina também ser utilizada. A vantagem da ecocardiografia sob estresse associada ao uso do contraste é a associação da análise da perfusão e da contração, no mesmo momento e no mesmo exame, de modo não invasivo, aumentando assim a sensibilidade, sem perda na especificidade do método, estando isento de material radioativo ou toxicidade ${ }^{11}$.

Quanto à análise do fluxo de reserva coronariano (FRC), este equivale a uma medida quantitativa da isquemia miocárdica, apresentando íntima relação com a perfusão miocárdica. O FRC é um importante indicador de gravidade da estenose epicárdica ou, na ausência de estenose, da integridade da microcirculação. Sua importância está na avaliação das consequências hemodinâmicas da lesão coronariana, de modo que essa análise deve fazer parte dos testes ecocardiográficos para pesquisa de isquemia miocárdica ${ }^{11}$.

O FRC corresponde à capacidade máxima de aumento do fluxo que pode ocorrer em um único vaso coronariano. $\mathrm{O}$ fluxo é analisado do seu ponto máximo (que pode ser obtido com a administração de vasodilatadores, como adenosina ou dipiridamol) em relação ao basal, sendo uma relação $>2,0$ considerada normal' ${ }^{12}$.

A sensibilidade e especificidade para detecção de obstrução $>75 \%$ na artéria descendente anterior, com um fluxo de reserva $<2,0$, é de $91 \%$ e $76 \%$, respectivamente ${ }^{11}$. A obtenção do FRC da artéria circunflexa e coronária direita é mais complexa, mas também pode ser feita com o uso do contraste.

A detecção de um fluxo de reserva $\geq 2,0$ é seguro para considerar baixa frequência de eventos cardíacos ${ }^{12}$.
Tabela 26 - Recomendações da ecocardiografia sob estresse na DAC crônica

\begin{tabular}{|c|c|}
\hline Recomendações & Classe \\
\hline Estratificação de risco de pacientes com DAC & I \\
\hline $\begin{array}{l}\text { Estresse farmacológico na avaliação de isquemia miocárdica em } \\
\text { indivíduos com precordialgia típica estável que não podem realizar } \\
\text { teste ergométrico máximo ou quando o teste ergométrico não é } \\
\text { diagnóstico }\end{array}$ & 1 \\
\hline $\begin{array}{l}\text { Avaliação de isquemia miocárdica em indivíduos assintomáticos } \\
\text { com teste ergométrico positivo ou duvidoso }\end{array}$ & I \\
\hline $\begin{array}{l}\text { Estresse farmacológico na avaliação pré operatória de cirurgia não } \\
\text { cardíaca de pacientes com três ou mais fatores de risco para DAC, } \\
\text { e que não podem se exercitar }\end{array}$ & I \\
\hline $\begin{array}{l}\text { Avaliação do significado funcional de lesões coronárias no } \\
\text { planejamento de angioplastia transluminal percutânea ou cirurgia } \\
\text { de revascularização }\end{array}$ & I \\
\hline $\begin{array}{l}\text { Avaliação de isquemia miocárdica na presença de bloqueio de } \\
\text { ramo esquerdo ou alterações que impeçam adequada análise } \\
\text { eletrocardiográfica da isquemia }\end{array}$ & I \\
\hline $\begin{array}{l}\text { Estresse farmacológico na avaliação de viabilidade miocárdica } \\
\text { (miocárdio hibernado) para planejamento de revascularização }\end{array}$ & I \\
\hline $\begin{array}{l}\text { Avaliação de reestenose após revascularização em pacientes com } \\
\text { recorrência de sintomas típicos }\end{array}$ & Ila \\
\hline $\begin{array}{l}\text { Diagnóstico de isquemia miocárdica em pacientes selecionados } \\
\text { com baixa probabilidade pré-teste para DAC }\end{array}$ & Ila \\
\hline $\begin{array}{l}\text { Diagnóstico de isquemia miocárdica em pacientes selecionados } \\
\text { com alta probabilidade pré-teste para DAC }\end{array}$ & $\| \mathrm{lb}$ \\
\hline $\begin{array}{l}\text { Substituição rotineira do teste ergométrico em pacientes nos quais } \\
\text { a análise eletrocardiográfica é adequada }\end{array}$ & III \\
\hline Avaliação de rotina em pacientes assintomáticos após revascularização & III \\
\hline
\end{tabular}

Tabela 27 - Recomendações da ecocardiografia com contraste na DAC crônica

\begin{tabular}{lc}
\hline Recomendações & Classe \\
\hline $\begin{array}{l}\text { Eco com contraste para avaliação da função do VE global e } \\
\text { segmentar em pacientes com imagens subótimas }\end{array}$ & । \\
\hline $\begin{array}{l}\text { Eco sob estresse com contraste para delineamento de bordos } \\
\text { endocárdicos em pacientes com imagens subótimas em repouso }\end{array}$ & I \\
\hline Avaliação do fluxo de reserva coronariano* & lib \\
\hline $\begin{array}{l}\text { Eco com contraste para avaliação da perfusão miocárdica e } \\
\text { determinação da área infartada e prognóstico da função do VE }\end{array}$ & lib \\
\hline $\begin{array}{l}\text { Eco com contraste com avaliação da perfusão miocárdica para } \\
\text { determinação de isquemia e viabilidade miocárdica }\end{array}$ & III \\
\hline $\begin{array}{l}\text { Uso rotineiro do contraste ecocardiográfico em todos os pacientes } \\
\text { para delineamento de bordas endocárdicas }\end{array}$ & \\
\hline *Análise usualmente feita em conjunto com a ecocardiografia de estresse.
\end{tabular}




\section{Diretrizes}

\section{Referências}

1. Sicari R, Nihoyannopoulos P, Evangelista A, Kasprzak J, Lancellotti P, Poldermans D, et al; European Association of Echocardiography. Stress echocardiography expert consensus statement: European Association of Echocardiography (EAE). Eur J Echocardiogr. 2008; 9 (4): 415-37.

2. Douglas PS, Khandheria B, Stainback RF, Weissman NJ, Peterson ED, Hendel RC, et al. ACCF/ASE/ACEP/AHA/ASNC/SCAI/SCCT/SCMR 2008 appropriateness criteria for stress echocardiography. Circulation. 2008; 117: 1478-97.

3. Camarozano AC, Resende P, Siqueira-Filho AG, Weitzel LH, Noe R. The effects of beta-blockers on dubutamine-atropine stress echocardiography: early protocol versus standard protocol. Cardiovascular Ultrasound. 2006; 4: 30-4.

4. McCully RB, Roger VL, Mahoney DW, Karon BL, Oh JK, Miller FA, et al. Outcome after normal exercise echocardiography and predictors of subsequent cardiac events: follow-up of 1.325 patients. J Am Coll Cardiol. 1998; 31 (1): 144-9.

5. La Canna G, Alfieri O, Giubbini R, Gargano M, Ferrari R, Visioli O. Echocardiography during infusion of dobutamine for identification of reversible dysfunction in patients with chronic coronary artery disease. J Am Coll Cardiol. 1994; 23: 617-26.

6. Poldermans D, Fioretti PM, Forster T, Thomson IR, Boersma E, el-Said EM, et al. Dobutamine stress echocardiography for the assessment of perioperative cardiac risk in patients undergoing major non-cardiac vascular surgery. Circulation. 1993; 87: 1506-12.

\section{Avaliação de fontes emboligênicas}

O sucesso do tratamento ou a redução das sequelas na fase aguda do acidente vascular encefálico isquêmico (AVEI) depende fundamentalmente do reconhecimento precoce da doença, da extensão do processo e do diagnóstico etiológico correto. É importante enfatizar que se identifiquem situações que possam mimetizar AVEI e necessitem intervenção imediata (hipoglicemia, encefalopatia hipertensiva, convulsão etc.), para se evitar, em tais circunstâncias, medidas terapêuticas inapropriadas ${ }^{1}$. Tanto a prevalência como a incidência do AVEI aumentam

Tabela 28 - Pacientes sob risco de doença cardioembólica

\begin{tabular}{ll}
\hline Risco maior (provável) & Risco menor (possível) \\
\hline Fibrilação atrial & Forame oval patente \\
\hline Trombos intracavitários & Aneurisma do septo interatrial \\
\hline Estenose da valva mitral & Comunicação interatrial \\
\hline Prótese valvar aórtica ou mitral & Prolapso da valva mitral \\
\hline Endocardite infecciosa & Contraste espontâneo \\
\hline Infarto agudo do miocárdio & Strands na valva mitral \\
\hline Cardiomiopatia dilatada & Calcificação do anel mitral \\
\hline Mixoma atrial esquerdo & Calcificação valvular aórtica \\
\hline Placas ulceradas na aorta & Endocardite marântica \\
\hline
\end{tabular}

7. Mathias W, Arruda AL, Andrade JL, Campos O, Porter TR. Endocardial border delineation during dobutamine infusion through use of contrast echocardiography. Echocardiography. 2002; 19: 1-6.

8. Dolan MS, Kamal R, El-Shafei A, Puri S, Tamirisa K, Bierig M, et al. Effect of intravenous contrast for left ventricular opacification and border definition on sensitivity and specificity of dobutamine stress echocardiography compared with coronary angiopraphy in technically difficult patients. Am Heart J. 2001; 142: 908-15.

9. Dolan MS, Gala SS, Dodla S, Abdelmoneim SS, Xie F, Coutier D, et al. Safety and efficacy of commercially available ultrasound contrast agents for rest and stress echocardiography: a multicenter experience. J Am Coll Cardiol. 2009; 53: 32-8.

10. Plana JC, Mikati IA, Dokainish H, Lakkis N, Abukhalil RT, Davis R, et al. A randomized cross-over study for evaluation of the effect of image optimization with contrast on the diagnostic accuracy of dobutamine echocardiography in coronary artery disease: the OPTIMIZE trial. J Am Coll Cardiol. 2008; 1: 153-5.

11. Burns PN. Contrast imaging for echocardiography. Principles and instrumentations. Handbook of contrast echo: left ventricular function and myocardial perfusion. 2000; 133-47.

12. Chamuleau SA, Tio RA, De Cock CC, de Muinck ED, Pijls NHJ, van Eck-Smit BLF, et al. Prognostic value of coronary blood flow velocity and myocardial perfusion in intermediate coronary narrowings and multivessel disease. J Am Coll Cardiol. 2002; 39 (5): 852-8.

com a idade e comorbidades associadas. Dados recentes sugerem uma maior incidência de AVEI sucedendo o ataque isquêmico transitório (AIT), principalmente na primeira semana. O risco precoce de AVEI depois de AIT varia de 15\%-20\% em 90 dias $^{2}$. Diretrizes mais recentes aconselham que a avaliação e o manuseio dos pacientes com AIT deveriam ocorrer dentro de uma ou duas semanas do evento, na tentativa de se prevenir o risco de futuro $\mathrm{AVEI}^{3}$.

\section{1 - Doença cardioembólica}

O evento neurológico cardioembólico é definido quando, na ausência de doença cerebrovascular em paciente com AVEI não lacunar, uma potencial fonte cardioembólica é identificada. A evidência de AIT prévio ou AVEI em mais de um território vascular ou embolia sistêmica corrobora o diagnóstico clínico de AVEI cardioembólico. Considerando-se estabelecido o diagnóstico de AVEI ou AIT, a literatura mostra um percentual de cerca de $20 \%$ quando se trata de fonte cardioembólica. Essa prevalência pode atingir 40\% quando se inclui os AVEI criptogênicos, podendo atingir até 50\% em pacientes mais jovens ${ }^{4}$.

Nesses cenários, o ETT e ETE tornam-se indispensáveis para identificar a etiologia da doença cardioembólica. Embasados em estudo retrospectivo, alguns autores discordam da utilidade da ETE como recurso de rotina diagnóstica nos pacientes com mais de 65 anos de idade ${ }^{5}$. Análise multivariada ajustada para idade, espessamento da placa ateromatosa, presença de doença coronária e hipertensão arterial identificaram o forame oval patente 